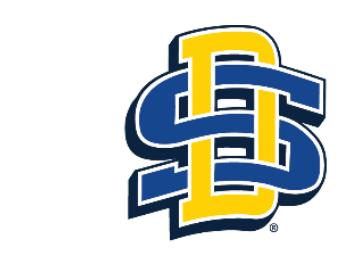

SOUTH DAKOTA STATE UNIVERSITY

Department of Animal Science

\section{Beef Day 2020}

Meats \& Human Nutrition

\title{
Influence of maternal protein restriction in primiparous heifers during mid-and/or late-gestation on meat quality and fatty acid profile of progeny
}

M.J. Webb, J.J. Block, R.N. Funston, K.R. Underwood, J.F. Legako, A.A. Harty, R.R. Salverson, K.C. Olson, and A.D. Blair

\section{Objective}

The objective of this study was to evaluate the influence of metabolizable protein (MP) restriction in mid- and/or late-gestation on meat quality, fatty acid profile, and carcass composition of progeny.

\section{Study Description}

Heifers were assigned to 2 levels of dietary protein (control [CON], 102\% of MP requirements; or restricted [RES], $80 \%$ of MP requirements) at 2 stages of gestation (mid-gestation [MID] and late-gestation [LATE]) in a Balaam's Design crossover treatment structure resulting in 4 treatment combinations (CON-CON, CON-RES, RES-CON, RES-RES). After calving, cow-calf pairs were moved to pasture and managed as a common group through weaning. Following weaning, calves were finished in a GrowSafe feeding system to a common backfat endpoint. At harvest standard carcass data was collected, lean color $\left(L^{*}, a^{*}, b^{*}\right)$ was determined, and strip loins were collected for and fabricated into 1 -inch steaks for determination of crude fat and tenderness at 3, 7, 14, and 21 days of aging using the Warner-Bratzler shear force method. An additional steak from a subsample of steer progeny $(n=24)$ was aged 3 days postmortem, frozen, and used for direct fatty acid methyl ester synthesis (determination of fatty acid profile). The 9-10-11 rib section of this same subsample of carcasses were removed from the left side of each carcass to determine carcass composition.

Take home points

A carryover effect of MID MP treatment on LATE CON indicated CON-CON steaks were more tender $(P<0.001)$ than RES CON. Mid-gestation restriction resulted in progeny with increased $(P<0.05)$ carcass water, soft tissue moisture, and decreased soft tissue fat percentage compared with progeny from dams receiving MID CON. Maternal MP restriction in both MID and LATE influenced fatty acid composition of steaks from progeny. Progeny from dams in this study were not subjected to any additional nutrient restrictions beyond gestational treatments; therefore, it is possible progeny were able to overcome a moderate MP restriction during development with adequate postnatal nutrient intake. Alternatively, dams may have mobilized protein reserves to buffer against the MP restriction imposed. 


\section{Acknowledgements}

Financial support of this project was provided by the Beef Checkoff through the South Dakota Beef Industry Council (Award\# 3X4504) and by state and federal funds appropriated to South Dakota State University including support by the USDA National Institute of Food and Agriculture, Hatch project (accession no. 1020088).

Keywords: beef, carcass composition, fatty acid, tenderness 


\title{
Influence of maternal protein restriction in primiparous heifers during mid- and/or late- gestation on meat quality and fatty acid profile of progeny
}

\author{
M.J. Webb, J.J. Block, R.N. Funston, K.R. Underwood, J.F. Legako, A.A. Harty, R.R. Salverson, K.C. \\ Olson, and A.D. Blair
}

\section{Abstract}

The objective of this study was to evaluate the influence of metabolizable protein (MP) restriction in mid-and/or late-gestation on meat quality characteristics of progeny. Heifers were assigned to 2 levels of dietary protein (control [CON], 102\% of MP requirements; or restricted [RES], $80 \%$ of MP requirements) at 2 stages of gestation (mid-gestation [MID] and late-gestation [LATE]) in a Balaam's Design crossover treatment structure resulting in 4 treatment combinations (CON-CON, CON-RES, RES-CON, RES-RES). A carryover effect of MID MP treatment on LATE CON indicated CON-CON steaks were more tender $(P<0.001)$ than RES CON. Mid-gestation restriction resulted in progeny with increased $(P<0.05)$ carcass water, soft tissue moisture, and decreased soft tissue fat percentage compared with progeny from dams receiving MID CON. Reduced maternal MP also differentially influenced the fatty acid profiles of progeny. Results suggest it is possible for progeny to overcome a moderate gestational MP restriction with minimal impacts on carcass composition or meat characteristics.

\section{Introduction}

Nutrient status of cows can vary throughout gestation and is subject to changes in forage availability, quality, and access to supplemental feedstuffs (DelCurto et al., 2000). Changes in maternal nutrient status can have long-term implications on progeny growth, feed efficiency, muscle development, and ultimately meat quality (Du et al., 2010; Funston et al., 2010; Wilson, 1999). Fetal skeletal muscle and fat cell development is primarily initiated in the second trimester (Du et al., 2010), at a time when fluctuations in forage quality and/or quantity can result in restricted nutrient intake in gestating cows (DelCurto et al., 2000). Any changes in the development and inherent properties of muscle and/or fat could result in a lasting impact on body composition and ultimately meat quality characteristics. Protein is often the first limiting nutrient for gestating beef cows consuming low-quality forage-based diets, and protein restriction can influence energy status (Cochran et al., 1998). The objective of this study was to evaluate the impacts of maternal protein restriction in mid-gestation, late-gestation, or throughout mid- and late-gestation on meat quality, carcass composition, and fatty acid (FA) profile of offspring.

\section{Experimental Procedures}

One hundred eight commercial crossbred Angus $x$ Simmental primiparous heifers were allocated to 12 open feedlot pens at the SDSU Cottonwood Range and Livestock Research Station. Rectal ultrasonography was conducted in mid-September of 2013 for pregnancy detection and sex and age of the fetus. Heifers were assigned to blocks in a randomized complete block design according to body weight (BW) and method of conception (artificial insemination vs. natural service). After blocking, heifers were assigned to pens in order to equalize the number of male and female offspring in each pen. This blocking structure resulted in 3 blocks with 4 pens per block. A Balaam's Design crossover treatment structure was used to 
apply 2 levels of dietary metabolizable protein (MP): control (CON, receiving approximately $102 \%$ of MP requirements) and restricted (RES, receiving approximately $80 \%$ of MP requirements) during each of 2 periods; mid-gestation (MID, d 148 to $d 216$ of gestation based on estimated date of conception) and late-gestation (LATE, $d 217$ of gestation to parturition). The resulting 4 treatment combinations were mid-gestation MP control followed by late gestation MP control (CON-CON), mid-gestation MP control followed by late gestation MP restricted (CON-RES), mid-gestation MP restricted followed by late gestation MP control (RESCON), and mid-gestation MP restricted followed by late gestation MP restricted (RES-RES). These 4 treatments combinations were randomly assigned to a pen within each block, resulting in 3 pen replicates per treatment combination. Diets consisted of calcium hydroxide treated wheat straw, crude glycerin, and concentrates. Treatment diets were isocaloric within gestation period and met NRC net energy requirements (2000) for maintenance, growth and pregnancy. Wheat straw was treated with $\mathrm{Ca}(\mathrm{OH})_{2}$ using the SecondCrop ${ }^{\mathrm{TM}}$ process (ADM Animal Nutrition, Quincy, IL) to increase energy value. Gestating heifers were delivered their assigned ration once per day beginning in early November 2013 until parturition in the spring of 2014. Heifer body weight (BW) and body condition score data were collected at trial initiation, at treatment crossover, and approximately 3 weeks prior to calving. Mean heifer BW for CON and RES heifers was 431 and $418 \mathrm{~kg} \pm 8.6$ and mean BCS was 4.9 and $4.8 \pm 0.05$, respectively, during mid-gestation. In late gestation, mean heifer BW for CON and RES heifers was 451 and $445 \mathrm{~kg} \pm 8.6$ and mean BCS was 4.8 and $4.7 \pm 0.05$, respectively. Animal performance indicated that diets met targets based on nutrients supplied. After calving, cow-calf pairs were moved to pasture with a common level of nutrition for spring calving cows and managed as a common group through weaning. All progeny were fed and managed under the same conditions from birth until harvest so the influence of gestational treatment could be determined. Steer and heifer progeny ( $n=103$ ) were backgrounded for $14 \mathrm{~d}$ after weaning at the SDSU Cottonwood Range and Livestock Research Station then transported to the University of Nebraska-Lincoln West Central Research and Extension Center, North Platte, NE, and adapted to a common finishing diet ( $48 \%$ dry rolled corn, $7 \%$ grass hay, $40 \%$ corn gluten feed, and $5 \%$ finisher supplement containing vitamin and minerals). Cattle were fed and managed to maintain health and achieve a common backfat thickness prior to harvest.

Cattle were transported to Tyson Fresh Meats, Lexington, NE for harvest. The Al-bred progeny were harvested 21 days earlier than the natural service bred progeny, which reflects the approximate difference in their birth dates. Actual mean backfat thickness was $1.37 \mathrm{~cm}$ and $1.34 \mathrm{~cm}$ for Al-bred and natural service groups, respectively. At harvest standard carcass data was collected, lean color $\left(L^{*}, a^{*}, b^{*}\right)$ was determined, and strip loins were collected for and fabricated into 1-inch steaks for determination of crude fat and tenderness at 3, 7, 14, and 21 days of aging. An additional steak from a subsample of steer progeny ( 6 steers per treatment combination) selected from artificially inseminated heifers of moderate body condition score and BW was aged $3 \mathrm{~d}$ postmortem, frozen, and used for direct fatty acid methyl ester (FAME) synthesis. The 9-10-11 rib section of this same subsample of carcasses were removed from the left side of each carcass to determine carcass composition. 


\section{Results and Discussion}

Meat color $\left(L^{*}, a^{*}, b^{*}\right)$, percent ether extractable fat and percent cook loss were not influenced by gestational treatment $(P>0.05)$. A MID $x$ LATE MP treatment interaction $(P<0.001)$ was detected for WBSF. Interactions in Balaam's Design indicate that the first period (MID) caused carryover effects on the second period (LATE). In this case, the contrasts indicated that MID MP treatments had carryover effects on LATE CON $(P<0.001)$, but not LATE RES $(P>0.05)$. The MID MP carryover on LATE CON contrast resulted because progeny of dams exposed to MID RES produced less tender steaks than MID CON, indicating restriction in mid-gestation (MID RES) followed by increased availability of MP (LATE CON) may alter the mechanisms involved in meat tenderness. Nonetheless, the WBSF values in the current study ranged from 26.9 to $31.9 \mathrm{~N}$. Miller et al. (2001) determined untrained consumers' perceived steaks to be tough at $45.1 \mathrm{~N}$ and tender at $42.2 \mathrm{~N}$. While all samples in this study would likely be considered tender by consumers, the average detected difference in WBSF value was $3.5 \mathrm{~N}$ between dams on the RES-CON treatment (31.9 N) and all other treatments $(28.4 \mathrm{~N})$. Underwood et al. (2010) evaluated dams grazing either improved pasture (6.0 - 11.1\% crude protein) or native range (5.4-6.5\% crude protein) from mid-gestation (d 120-150) through the beginning of the third trimester (d $180-210)$. Similar to results from the current study, maternal protein availability influenced progeny steak tenderness.

To date, evaluation of the effects of maternal diet on the fatty acid composition of progeny have not been studied (Greenwood et al., 2017). In this study myristic acid (C14:0) displayed a treatment by period interaction $(P<0.05)$. Contrasts revealed that the percentage of C14:0 in progeny samples from dams restricted LATE was reduced $(P<0.05)$ when dams were provided RES diet during MID (2.87\%) compared with dams receiving CON during MID (3.25\%). This indicates a carryover effect in which MID-RES followed by LATE-RES depressed deposition of $\mathrm{C} 14: 0$ in progeny steaks. When samples were evaluated on a $\mathrm{mg} / \mathrm{g}$ of raw wet tissue basis, the concentrations of palmitic acid (C16:0), oleic acid (C18:1n9 cis), total saturated fatty acid (SFA), total monounsaturated fatty acid (MUFA) and total lipid displayed carryover effects from MID MP treatments on both LATE CON and LATE RES. The carryover effect for LATE CON indicated steaks from progeny from dams that received MID CON (CON CON) had elevated levels of each of these FA relative to MID RES (RES CON). The carryover effect for LATE RES indicated the opposite wherein steaks from progeny from dams receiving MID CON (CON RES) had depressed levels of each of these relative to MID RES (RES RES). The concentration of arachidonic acid $(C 20: 4 n 6)$ was increased $(P<0.05)$ in progeny from LATE CON dams when provided MID CON, however it also increased $(P<0.05)$ when the LATE RES treatment followed MID CON. Despite these carryover effects for some individual FA and categories, MID RES often decreased FA concentrations ( $\mathrm{mg} / \mathrm{g}$ ) compared to MID CON, while LATE RES often increased FA concentrations relative to LATE-CON as indicated by main effect responses. Results indicate maternal MP restriction in both MID and LATE can influence FA composition of steaks from progeny and warrants further investigation.

The composite 9-10-11 rib sum for carcass composition and edible portions in this study ranged from $99.3 \%$ to $100.5 \%$. Percent carcass water was increased $(P<0.05)$ in progeny carcasses from dams receiving MID RES compared with MID CON. Evaluation of soft tissue responses 
indicated that dams receiving MID RES resulted in increased $(P<0.05)$ progeny soft tissue moisture percentage and reduced $(P<0.05)$ fat percentage in the tissue. Soft tissue protein displayed a MID by LATE treatment interaction $(P<0.05)$. Percent protein in progeny samples from dams receiving LATE CON was decreased $(P<0.001)$ when dams received MID RES compared to MID CON, whereas protein percentage of progeny from dams receiving LATE RES was increased $(P<0.001)$ when dams received MID RES compared to MID CON. The thrifty phenotype hypothesis suggests that maternal dietary insults during fetal development can result in developmental changes that 'program' the offspring to adapt to the environment it is born into. Perhaps progeny that experienced a gestational protein restriction were programmed to conserve protein tissue. Although differences in soft tissue composition were detected, these differences were small and may not have biological significance. Evaluation of maternal nutrition effects on beef carcass composition is limited, however, Underwood et al. (2010) also reported increased percent moisture and decreased percent crude fat in the LM from progeny of dams grazing native range (limited protein) compared with progeny of dams grazing improved pasture.

\section{Implications}

Reduced maternal MP in mid-gestation decreased tenderness of steaks from progeny, although late-gestation maternal MP restriction mitigated this effect. Reduced maternal MP in both midand late gestation altered FA profile of progeny steaks, with mid-gestation MP restriction often increasing FA content and late-gestation MP restriction often decreasing FA content in progeny steaks. Mid-gestation and/or late-gestation periods of maternal MP restriction did not negatively influence beef color, percent cook loss, or ether extractable fat. Mid-gestation MP restriction shifted soft tissue composition toward increased protein and decreased fat content, but the differences were minor and may not be biologically relevant. Progeny from dams in this study were not subjected to any additional nutrient restrictions beyond gestational treatments; therefore, it is possible progeny were able to overcome a moderate MP restriction during development with adequate postnatal nutrient intake. Alternatively, dams may have mobilized protein reserves to buffer against the MP restriction imposed.

\section{References}

Cochran, R.C., Koster, H.H., Olson, K.C., Heldt, J.S., Mathis, C.P., and Woods, B.C. 1998. Proc $9^{\text {th }}$ Florida Nutr. Symp. pp.123 - 136

DelCurto, T. B., Hess B. W., Huston J. E., and Olson, K. C. 2000. J Anim. Sci. 77:1-16

Du, M., Tong, J., Zhao, J., Underwood, K.R., Zhu, M., Ford, S.P, and Nathanielsz, P.W. 2010. J Anim. Sci. 88:E51-E60

Funston, R.N., Larson D.M., and Vonnahme, K.A. 2010. J Anim. Sci. 88:E205-E215

Greenwood, P., Clayton, E. and Bell A. 2017. Anim. Front. 7:38-47

Miller, M.F., Carr, M.A., Ramsey, C.B., Crockett, K.L., and Hoover, L.C. 2001. J Anim. Sci. 79:3062-3068

Nutrient Requirements of Beef Cattle (NRC) $7^{\text {th }}$ Ed. 2000. Washington, DC: National Academy Press

Underwood, K.R., Tong, J.F., Price, P.L., Roberts, A.J., Grings, E.E., Hess, B.W., Means, W.J., and Du, M. 2010. Meat Sci. 86:588-593 
Wilson, J. 1999. The Australian and New Zealand J of Obstet Gynaecol. 39:1-7 